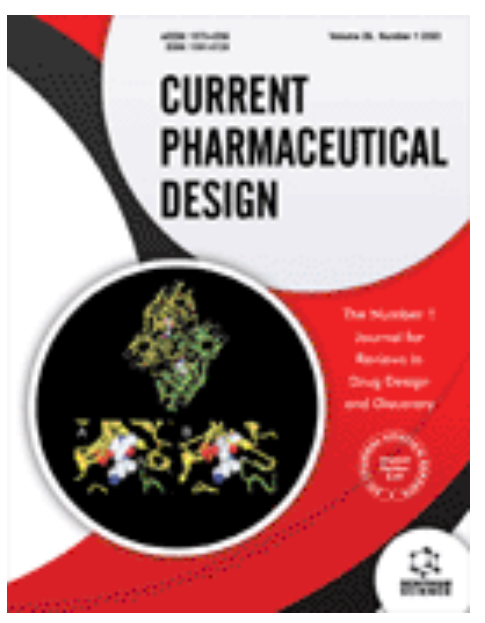

Research Article

\title{
Covid-19 And Rheumatic Autoimmune Systemic Diseases: Role of Pre-Existing Lung Involvement and Ongoing Treatments
}

(E-pub Abstract Ahead of Print)

Author(s): Clodoveo Ferri, Dilia Giuggioli, Vincenzo Raimondo, Massimo L'Andolina, Lorenzo Dagna, Antonio Tavoni, Francesco Caso, Francesco Ursini, Ruscitti Piero, Maurizio Caminiti, Rosario Foti, Valeria Riccieri, Serena Guiducci, Roberta Pellegrini, Elisabetta Zanatta, Giuseppe Varcasia, Domenico Olivo, Pietro Gigliotti, Giovanna Cuomo, Giuseppe Murdaca, Riccardo Cecchetti, Rossella De Angelis, Nicoletta Romeo, Francesca Ingegnoli, Franco Cozzi, Veronica Codullo, Ilaria Cavazzana, Michele Colaci, Giuseppina Abignano, Maria De Santis, Ennio Lubrano, Enrico Fusaro, Alessandra Della Rossa, Amelia Spinella, Federica Lumetti, Giacomo De Luca, Silvia Bellando-Randone, Elisa Visalli, Ylenia Dal Bosco, Giorgio Amato, Daiana Giannini, Silvia Bilia, Francesco Masini, Greta Pellegrino, Erika Pigatto, Elena Generali, Giuseppa Pagano Mariano, Giorgio Pettiti, Giovanni Zanframundo, Raffaele Brittelli, Vincenzo Aiello, Rodolfo Caminiti, Daniela Scorpiniti, Tommaso Ferrari, Corrado Campochiaro, Veronica Brusi, Micaela Fredi, Liala Moschetti, Fabio Cacciapaglia, Laura Gragnani, Monica Monti, Serena Lorini, Sabrina Rosaria Paparo, Francesca Ragusa, Valeria Mazzi, Giusy Elia, Silvia Martina Ferrari, Ilenia Di Cola, Marta Vadacca, Sebastiano Lorusso, Simone Barsotti, Maria Letizia Aprile, Tasso Marco, Mario Miccoli, Silvia Bosello, Marco Matucci-Cerinic, Salvatore D'Angelo, Andrea Doria, Franco Franceschini, Riccardo Meliconi, Florenzo lannone, Roberto Giacomelli, Anna Linda Zignego, Poupak Varcasia, Alessandro Antonelli*

Journal Name: Current Pharmaceutical Design

DOI : $10.2174 / 1381612827666210903103935$ 